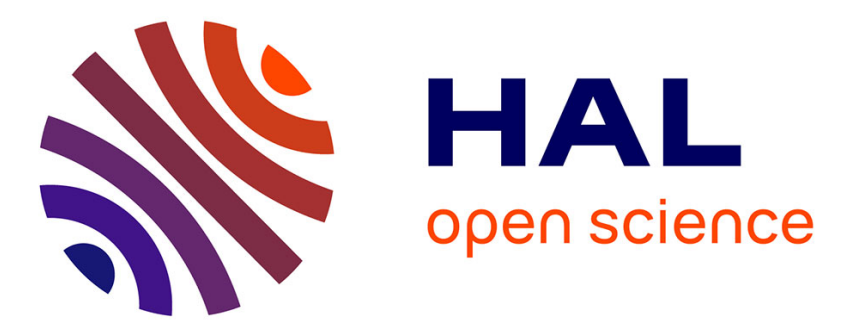

\title{
Premiers résultats du programme d'étude de l'Holocène de Tunisie
}

Jean-Louis Ballais, M. Bourgou, R. Karray, J.-P. Lautridou, M. Levant, Ameur Oueslati, H. Ben Ouezdou, T. Bou Hafa, Abderrazak Gragueb

\section{To cite this version:}

Jean-Louis Ballais, M. Bourgou, R. Karray, J.-P. Lautridou, M. Levant, et al.. Premiers résultats du programme d'étude de l'Holocène de Tunisie. Méditerranée: revue géographique des pays méditerranéens, 1988, 64 (2), pp.64 - 67. 10.3406/medit.1988.2552 . hal-01562383

\section{HAL Id: hal-01562383 \\ https://hal-amu.archives-ouvertes.fr/hal-01562383}

Submitted on 14 Jul 2017

HAL is a multi-disciplinary open access archive for the deposit and dissemination of scientific research documents, whether they are published or not. The documents may come from teaching and research institutions in France or abroad, or from public or private research centers.
L'archive ouverte pluridisciplinaire HAL, est destinée au dépôt et à la diffusion de documents scientifiques de niveau recherche, publiés ou non, émanant des établissements d'enseignement et de recherche français ou étrangers, des laboratoires publics ou privés. 


\section{Jean-Louis Ballais}

M. Bourgou

R. Karray

J.P. Lautridou

M. Levant

Ameur Oueslati

H. Ben Ouezdou

T. Bou Hafa

Abderrazak Gragueb

\section{Premiers résultats du programme d'étude de l'Holocène de}

\section{Tunisie}

In: Méditerranée, Troisième série, Tome 64, 2-1988. Évolution des paysages tunisiens au cours du Quaternaire. pp. 64-67.

\section{Abstract}

First studies have established that one holocene terrace and one historic terrace have been accumulated. Fills of natural or anthropic dams also exist. Confirmation has been brought about the existence of pre-Capsian blown sands, lower to middle Holocene gypcretes and two post-Versilian marine transgressions.

\section{Résumé}

Les premières recherches ont établi l'importance de la dynamique fluviatile qui a accumulé une terrasse holocène s. s. et une terrasse historique, sans compter les accumulations à l'amont de barrages naturels ou artificiels. Elles ont confirmé l'existence de dépôts éoliens pré-capsiens, de croûtes gypseuses de l'Holocène inférieur-moyen et de deux remontées relatives du niveau marin depuis le Versilien.

Citer ce document / Cite this document :

Ballais Jean-Louis, Bourgou M., Karray R., Lautridou J.P., Levant M., Oueslati Ameur, Ben Ouezdou H., Bou Hafa T., Gragueb Abderrazak. Premiers résultats du programme d'étude de l'Holocène de Tunisie. In: Méditerranée, Troisième série, Tome 64, 21988. Évolution des paysages tunisiens au cours du Quaternaire. pp. 64-67.

doi : 10.3406/medit.1988.2552

http://www.persee.fr/web/revues/home/prescript/article/medit_0025-8296_1988_num_64_2_2552 


\section{Premiers résultats du programme d'étude de l'Holocène de Tunisie}

\author{
J.-L. BALLAIS* \\ M. BOURGOU* \\ R. KARRAY* \\ J.P. LAUTRIDOU* * \\ M. LEVANT** \\ A. OUESLATI* \\ H. BEN OUEZDOU*** \\ T. BOU HAFA* \\ A. GRAGUEB ****
}

Résumé - Les premières recherches ont établi l'importance de la dynamique fluviatile qui a accumulè une terrasse holocène s.s. et une terrasse historique, sans compter les accumulations à l'amont de barrages naturels ou artificiels. Elles ont confirmé l'existence de dépôts éoliens pré-capsiens, de croûtes gypseuses de l'Holocène inférieur-moyen et de deux remontées relatives du niveau marin deputs le Versilien.

Abstract - First studies have established that one holocene terrace and one historic terrace have been accumulated. Fills of natural or anthropic dams also exist. Confirmation has been brought about the existence of pre-Capsian blown sands, lower to middle Holocene gypcretes and two post-Versilian marine transgressions.

L'étude de l'Holocène de Tunisie fait l'objet d'un programme de recherche de la Faculté des Sciences Humaines et Sociales de Tunis qui regroupe les géomorphologues de cet établissement (Jean-Louis Ballais, Mongi Bourgou, Raouf Karray et Ameur Oueslati) en collaboration avec le Centre de Géomorphologie du C.N.R.S. de Caen (Jean-Pierre Lautridou, Michèle Levant) et le Laboratoire d'Hydrologie et de Géochimie isotopique de Paris-Orsay (Jean-Charles Fontes). Les premières recherches ont tendu à établir un inventaire aussi exhaustif que possible des formes et formations holocènes en Tunisie, en s'appuyant sur une bibliogra. phie déjà abondante, ancienne (1) ou récente (HAMZA, 1983 ; OUESLATI, 1985 ; BEN OUEZDOU, 1986 ; BOURGOU et OUESLATI, 1987) et sur nos recherches actuelles. Elles ont abouti à la reconnaissance de leur complexité et de l'importance de la dynamique fluviatile, mais aussi à la confirmation de l'existence de formations originales, à l'Holocène inférieur-moyen, ainsi que de fluctuations du niveau marin.

\section{I - LES FORMES ET FORMATIONS LIEES A LA DYNAMIQUE FLUVIATILE}

Le long des cours d'eau de Tunisie, le nombre des accumulations et banquettes holocènes s. 1 . est très variable, en général de 1 à 4 , mais c'est, en fait, six formes différentes qu'il faut parfois distinguer selon des critères chronologiques.

\section{Les terrasses et autres accumulations holocènes s.1.}

Certaines sont attribuées à l'Holocène parce qu'elles contiennent des industries préhistoriques antérieures (ou contemporaines, au plus jeune) remaniées : industries ibéromaurusienne, épipaléolithique indiffé. renciée, capsienne ou néolithique (11 cas répartis du Nord au Sud).

D'autres sont attribuées à l'Holocène parce qu'elles sont emboîtées dans les formes ou formations du Pléistocène supérieur ou étagées en-dessous $(6 \mathrm{cas})$. Comme les précédentes, elles sont généralement de texture fine, de teinte brune à grise, grossières surtout au pied des reliefs. Elles présentent souvent des niveaux noirâtres, hydromorphes, parfois des concentrations diffuses de fer au Nord, de calcaire au Centre et de gypse au Sud.

* Faculté des Sciences Humaines et Sociales, 94, Bd du 9 Avril 1938, Tunis, - ** Centre de Géomorphologie du C.N.R.S., Caen, - *** Centre des Sciences de la Terre, I.N.R.S.T., B.P. 95, Hammam Lif, - **** Institut National d'Archéologie et d'Art, Tunis.

(1) qu'il n'est pas possible de citer, faute de place. 
Enfin, il faut signaler deux exemples, dans le Sud, de terrasses d'altitude relative aberrante par rapport aux prédédentes, attribuées à l'accumulation à l'amont d'un barrage naturel dont l'origine reste à préciser.

\section{Les terrasses et autres accumulations historiques}

On distingue très souvent une terrasse historique dans l'entaille de laquelle peuvent s'individualiser deux banquettes.

La terrasse historique, de teinte grise et de texture fine, contient toujours des tessons de céramique romaine ou plus ancienne ( 7 cas connus, du Nord au Sud) et souvent des charbons de bois, des foyers en place, voire des ossements. Elle peut s'inscrire directement dans les formations du Pléistocène supérieur, couvre une surface considérable et est constituée d'une accumulation épaisse de plusieurs mètres. C'est le kyounger fill» de C. VITA-FINZI (1969). Pour les plus grands organismes (O. Miliane, O. Maarouf, O. Leben), l'étude fine des figures de sédimentation montre des différenciations en fonction de la latitude.

La banquette supérieure, souvent étroite, vers 2 à $4 \mathrm{~m}$ au-dessus du lit mineur, peut se présenter comme une terrasse d'ablation aux dépens du matériel de la terrasse précédente, ou comme une accumulation de quelques décimètres, fine ou grossière. Dans un cas, elle contient des tessons de céramique islamique.

La banquette inférieure peut constituer une étroite terrasse, en particulier dans les lobes convexes de méandres, vers $1 \mathrm{~m}$ à $1,50 \mathrm{~m}$ au-dessus du lit mineur. Souvent assez grossière, parfois manifestement encore fonctionnelle lors de grandes crues, elle est parfois déjà fossile. Cette succession d'une terrasse et de deux banquettes a été observée dans six cas répartis du Nord au Sud de la Tunisie.

Le plus souvent (17 cas répertoriés), il n'existe qu'une seule terrasse contenant de la céramique romaine, peutêtre plus particulièrement dans le Nord du pays. Elle présente alors les caractéristiques de la terrasse historique ci-dessus, mais avec une texture grossière dès que les versants sont proches. Dans quelques cas, identifiés pour l'instant surtout dans le Sud, une basse terrasse de texture grossière peut être historique, dans la mesure où elle s'emboîte dans la terrasse holocène datée. Enfin, nous avons reconnu aussi trois cas de fausse terrasse dues à l'accumulation alluviale à l'amont de barrages artificiels romains. Cette accumulation, dont l'étude sera précieuse pour la connaissance de la morphogénèse durant cette période, présente des traits proches de ceux de la terrasse historique.

La reconnaissance de ces terrasses permet de comparer l'altitude actuelle du lit mineur des cours d'eau avec celle des constructions d'époque romaine (ponts, aqueducs, barrages surtout) montrant que, dans 9 cas, les cours d'eau retrouvant leur altitude de la période romaine (parfois même, plus précisément, du Ilème s. ap. J.C.) et que, dans 4 cas, l'entaille actuelle est plus profonde que l'entaille romaine de $0.50 \mathrm{~m}$ à $2 \mathrm{~m}$.

\section{II - LES FORMATIONS LIEES A LA DYNAMIQUE EOLIENNE}

\section{Elles constituent deux groupes distincts : les sables éoliens et des croûtes gypseuses.}

1. Les sables éoliens (photos 1 et 2) Photo 1

Photo 2
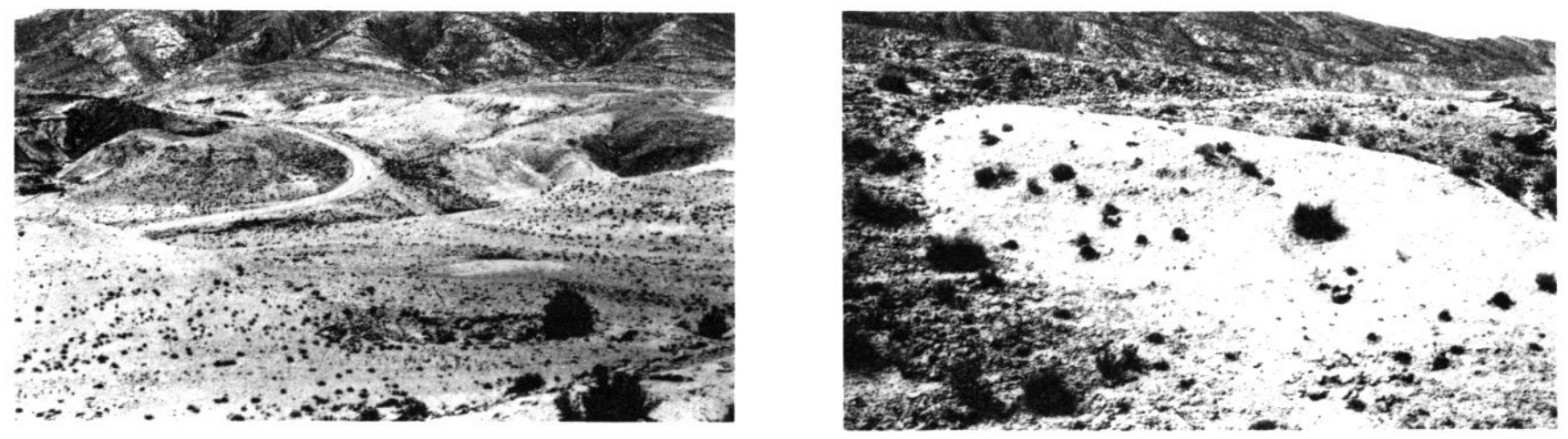

Photo 1 : PLACAGES DE SABLES EOLIENS A INDUSTRIE A LAMELLES SUR LE FLANC NORD DU DJEBEL UOM ALI - VUE GENERALE

Photo 2 : IDEM - VUE DE DETAIL

(Clichés : J.-L. Ballais) 
Ces sables éoliens reposent sur la terrasse du Pléistocène supérieur ou sur le substratum anté-quatemaire et sont scellés par un site capsien. Deux nouveaux exemples ont été reconnus, en plus de celui de l'oued el Akarit déjà signalé (GOBERT et HOWE, 1952 ; GOBERT, 1962 ; GRAGUEB, 1983). Ils confirment l'extension de la dynamique éolienne très au Nord de son aire actuelle, lors de l'Holocène inférieur (BALLAIS et BENAZ.ZOUZ, 1987).

\section{Les croûtes gypseuses}

Le deuxième groupe est constitué par des croûtes gypseuses :qui viennent s'ajouter à celle déjà reconnue à l'oued el Akarit, postérieure à $5595 \pm 500$ B.P. (FONTES et al., 1983) : une qui encroûte une escargotière capsienne et deux sur une terrasse contenant une industrie épipaléolithique dont une supportant des sites néolithiques. Comme le groupe précédent, il est localisé dans le Sud, mais de façon moins exclusive. Par contre, il serait prématuré de prétendre que toutes ces croûtes sont d'origine éolienne.

\section{III - LES FLUCTUATIONS DU NIVEAU MARIN}

Outre le maximum versilien de $+2 \mathrm{~m}$, daté de $5920 \pm 110$ B.P. par J.P. PERTHUISOT (1975), les côtes de la Tunisie méridionale renferment les témoins d'un deuxième niveau marin supérieur à l'actuel et daté, à Jerba, d'environ 2700 B.P. (DALONGEVILLE et al., 1980). Ce deuxième niveau pourrait exister aussi au Nord de Gabès où, vers $+1 \mathrm{~m}$, une falaise fossile entaille les loess datés de $3600 \pm 40$ et $3100 \pm 800$ B.P. (FONTES et al., 1983), mais n'affecte pas un établissement romain important qui paraît donc postérieur. Des variations du niveau de la mer au cours de l'Antiquité ont été également démontrées.C'est le cas, par exemple, dans le golfe de Tunis où, au cours de l'époque carthaginoise et romaine, le niveau marin se situait à quelques décimètres au-dessous du zéro actuel (PASKOFF et al., 1985 ; OUESLATI et al., 1987). Enfin, la destruction actuelle, sous le coup des vagues de la lagune de Bizerte, du site punique et romain de Jouaouada confirme une remontée relative du niveau marin depuis l'époque romaine.

\section{IV - CONCLUSION}

Confirmations de faits connus ou apports nouveaux, il ne s'agit encore que d'observations de terrain, pour l'essentiel, mais elles mettent en lumière la complexité de l'évolution des paysages au cours de l'Holocène et, surtout, de la période historique. Des hypothèses pourraient être avancées dès maintenant, mais la reconstitution des conditions précises de la genèse des formes et formations (morphogénèse, climat, néotectonique, actions anthropiques) ne pourra se faire qu'après toute une série d'analyses et de datations qui permettront de trancher entre cès différents hypothèses.

\section{SELECTION BIBLIOGRAPHIQUE}

BALLAIS J.-P. et BENAZZOUZ M.T. (1987). - Sédimentation quaternaire lacustre et éolienne dans le BasSahara algérien, 8 th Regional Mee ting of Sedimentology, Tunis, 54-55.

BEN OUEZDOU H. (1986).- Essai de corrélation des formations quaternaires continentales et marines dans les alentours du Golfe de Gabès, Géodynamique, 1, (2), ORSTOM, Paris, 3-17.

BOURGOU M. et OUESLATI A. (1987).- Les dépôts récents, en particulier ceux d'âge historique, de la vallée du Kébir-Miliane (Nord-Est de la Tunisie), Méditerrạée, Aix-en-Provence, $n^{\circ} 1$, 43-49.

DAlONGeVille R., PASKOFF R., SANLAVILlE P., THOMMERET J. et THOMMERET Y. (1980).Témoins d'un niveau marin holocène supérieur à l'actuel en Tunisie méridionale, C. R. Ac. Sc. Paris, t. 290, série D, 303-306.

FONTES J.-C., COQUE R., DEVER L., FILLY A. et MAMOU A. (1983).- Paléohydrologie isotopique de l'oued el Akarit (Sud Tunisie) au Pléistocène supérieur et à l'Holocène, Palaeog., Palaeocl., Palaeoecol., Elsevier Sc. Publ. B.V., Amsterdam, 41-62.

GOBERT E. G. (1962).- La préhistoire dans la zone littorale de la Tunisie, Quaternaria, VI, 271-307.

GOBERT E. G. et HOWE B. (1955).- L'Ibéromaurusien de l'oued El Akarit (Tunisie), Actes Ilème Cong. Panaf. Préhist., Alger, 1952, 575-598. 
GRAGUEB A. (1983).- Ibéromaurusien et Industries à lamelles en Tunisie; Thèse 3ème cycle, Aixen-Provence, 2 vol., 229 p. +196 fig.

HAMZA A. (1983).- Contribution à l'étude de la morphogénèse historique en Tunisie centrale : le cas du bassin versant de l'oued H'Jel, Rech. géogr. a Strasbourg, n²2-23, 55-77.

OUESLATI A. (1985).- Les dépôts holocènes liés au ruissellement sur les côtes de la Tunisie, Notes Serv. géol., Tunis, $\mathrm{n}^{\circ} 51$, sous presse.

OUESLATI A., PASKOFF R., SLIM H. et TROUSSET P. (1987).- Déplacements de la ligne de rivage en Tunisie d'après les données de l'archéologie à l'époque historique, Coll. Déplacements des lignes de rivage en Méditerranée, C.N.R.S., Paris, 67-85.

PASKOFF R., HURSTY H. et RAKOB F. (1985).- Position du niveau de la mer et déplacement de la ligne de rivage à Carthage (Tunisie) dans l'Antiquité, C. R. Ac. Paris, t. 300, II, 1, 3, 613-618.

PERTHUISOT J.P. (1975).- La Sebkha el Melah de Zarzis. Genèse et évolution d'un bassin paralique, Trav. du Lab. de Géol., n 9, E.N.S., Paris, 252 p.

VITA-FINZI C. (1969).- The Mediterranean Valleys. Geological Changes in Historical Times, Cambridge Univ. Press., 140 p. 